[post-print version]

\title{
Women's use of online resources and acceptance of e-mental health tools during the perinatal period
}

\author{
Ana Fonseca, Ricardo Gorayeb, \& Maria Cristina Canavarro
}

\begin{abstract}
Objectives: The women's professional help-seeking rate for perinatal depression is low, despite the prevalent and disabling nature of this condition. Therefore, new approaches should be implemented to increase women's access and utilization of treatment resources, namely e-mental health tools. This study aimed to characterize women's current pattern of use of online resources for mental heal issues and women's acceptance of e-mental health tools during the perinatal period, and to investigate its main determinants.
\end{abstract}

Methods: This study used an online cross-sectional survey that was completed by 546 women during the perinatal period.

Results: $31.3 \%$ had prior knowledge of websites targeting mental health illness. Women presenting an actual need for help (i.e., a positive screen for depression) reported greater use of online resources and greater engagement in e-health behaviors related to mental health $(d=.46$ to .61$)$, and being more accepting of e-mental health tools, particularly of informative websites. Women's perceptions concerning the emental tools were found to predict their intentions to use them.

Conclusions: The results seem to globally support Portuguese women's acceptance of e-mental health tools. To improve the level of acceptance, women should be involved as stakeholders in the development of new e-mental health tools and provided with specific information before their utilization.

Key-words: acceptance; e-mental health tools; perinatal period; perinatal depression; 


\section{Introduction}

There is widespread agreement regarding the potential benefits of e-health in facilitating citizens' access to healthcare [1,2]. The value of e-health is also recognized in the mental health field [3]. E-mental health includes the delivery or enhancement of mental health information and services through the internet and related technologies [4,5]. Therefore, e-mental health can include different types of tools, which address different areas of mental health service delivery. Informative websites are e-mental health tools targeting information provision about mental health problems. Concerning intervention, e-mental health tools include both symptom prevention and management programs (i.e., web-based psychological interventions, with or whithout therapist guidance, based on evidence-based face-to-face delivery models such as Cognitive Behavioural Therapy and Interpersonal Therapy) and online counseling (i.e., online psychological appointments with a qualified therapist). Moreover, online peer support groups are e-mental health tools aiming to reduce social isolation, and to provide opportunities for sharing information and practical advice about mental health $[3,6]$.

Perinatal depression is a prevalent clinical condition [7], with adverse effects on maternal wellbeing [8] and on children's development $[9,10]$. However, few women proactively seek professional assistance for their mental health problems during the first months post-birth, compromising access to treatment and its associated outcomes. Practical (limited access to healthcare, lack of time due to childcare responsibilities) and attitudinal (stigma) barriers were found to prevent women from seeking professional assistance during the perinatal period $[11,12]$.

The development of e-mental health tools to prevent and treat perinatal depression may be one effective way to improve women's access and utilization of mental healthcare and its outcomes [13-15]. In fact, research shows that women already use the internet for health (e.g., to seek information about pregancy, birthing and/or baby care [16]) and mental health issues during the perinatal period. Specifically, women use te internet to search for perinatal depressive symptoms [17], and to participate in online peer support groups to obtain health information and emotional support [18]. Moreover, when considering webbased interventions for perinatal depression, recently developed in different countries (e.g., Norway, United Kingdom, USA, Australia, [14, 19-22]), women report these tools as being helpful in managing mood 
changes [e.g., 19], useful and credible [e.g., 14]. However, there is also evidence of low engagement with these web-based tools. For example, on the efficacy study of the web-based intervention program for postpartum depression conducted by O'Mahen and cols., only 39\% of women adhered to the program, and a decrease of the number of session openings (dropout) was observed over time [21]. Moreover, on the pilot randomized controlled trial conducted by Barrera et cols., only $26.3 \%$ of the women logged-on the webbased preventive program for depression three or more times [19].

Consistently, despite public access to effective e-mental health interventions is growing [23], there is also evidence of poor implementation and low adoption rates for such interventions [24, 25]. The use of e-mental health tools may be dependent on several nonspecific intervention factors, including individuals' prior experience with e-health interventions and their acceptance of these interventions as part of daily care [24, 26-29], and this should be further explored in women during the perinatal period.

Acceptance is defined as an individual's psychological state with regard to his/her intended use of a particular tool/product [30], and it may be an important barrier to the individual's adherence to e-mental health tools. The Combined Technology Acceptance Model and Theory of Planned Behavior (C-TAMTPB $[31,32])$ constitutes an important framework to understand the variables that may affect acceptance. According to the C-TAM-TPB, one's acceptance of e-mental health tools may be inferred from the individual's intention to use e-mental health tools in times of need. Individual's intentions may be influenced by the individual's attitudes toward e-mental health tools, perceptions of behavioral control (perceptions of internal and external constraints on the use of the tool), the usefulness and ease of use of the e-mental health tool, and the individual's subjective norms (perceptions of a significant other's opinions concerning the use of e-mental health tools; [31, 32]).

Existing research with the general population shows that while some individuals rate e-mental health interventions as acceptable [38] and believe that they are effective in reducing psychopathological symptoms, others consider these tools only moderately acceptable concerning their helpfulness and credibility [24, 33]. Moreover, research shows that individuals endorse both positive and negative attitudes toward e-mental health tools, as they simultaneously perceive benefits (e.g., anonymity and reduced stigma, convenience of access, low treatment costs) and limitations (e.g., loss of therapeutic alliance, lack of privacy and security in interactions) of such tools $[24,34,35]$.

When comparing e-mental health tools with face-to-face treatments, the majority of individuals express more favorable attitudes concening face-to-face mental health services [24, 26, 36-37], and this 
may be explained by the individual's lower levels of knowedge and familiarity with e-mental health interventions $[37,38]$. Consistently, greater acceptance of e-mental health interventions was reported by previous users of such interventions $[38,39]$. However, it is also worth mentioning that in the study conducted by Klein et al. [38], only a minority (less than 10\%) of individuals reported complete unwillingness to use e-mental health services, and that they were globally available to learn more about emental health resources [26]. Moreover, there is some evidence that low levels of computer use and of ehealth literacy may influence individuals' acceptance of e-mental health tools [36, 40]. However, further research is needed to investigate how these factors play a role on women's acceptance of e-mental health tools during the perinatal period.

Furthermore, existing research focused on acceptance of e-mental health tools in general, failing to explore the individual's differences in acceptance, as a function of the type of e-mental health tools (e.g., informative websites, web-based psychological interventions, online psychological appointments, and online peer support groups). An exception is the study of Casey et al. [6], which found that informative websites were perceived as significantly more helpful and more likely to use than online psychological appointments and web-based psychological interventions. Moreover, the majority of the studies focused on the acceptance of e-mental health tools considering primarly individuals from the general population [e.g., $24,36,38]$. Few exceptions explored the acceptance of e-mental health tools by individuals already presenting psychopathological symptoms, that is, presenting an actual need for help [33]. To understand the acceptance of e-mental health tools - and the variables that may influence it - among women with an actual need for help during the perinatal period, is of particular relevance as they are the real target population of e-mental health tools.

Based on the previously mentioned literature gaps, this study focused on the perinatal period and aimed to: 1) describe women's current pattern of use of online resources for mental health issues (i.e., women's knowledge and utilization of online resources for health/mental health, informational and participatory e-health behaviors), and to examine differences in this pattern between women with an actual need for help (i.e., with a positive screening for depressive symptoms) and non-depressed women; 2) describe and compare women's acceptance (intentions to use) and acceptability-related perceptions (attitudes, subjective norms, perceived behavioral control, perceived usefulness and ease of use), as a function of different e-mental health tools (i.e., informative websites, web-based psychological interventions, online peer support groups and online psychological appointments) and of the presence of an 
actual need for help; and 3) investigate the effects of the prior use of online resources and of the acceptability-related perceptions in women's acceptance of different e-mental health tools, when they present an actual need for help.

\section{Materials and Methods}

\subsection{Procedure}

This study was part of a cross-sectional internet survey conducted in Portugal. The study followed the ethical standards and procedures for research with human beings (Helsinki Declaration - World Medical Association [41]; American Psychological Association [42]), and was approved by the Ethical Committee of the Faculty of Psychology and Educational Sciences of University of Coimbra. The participants constituted a self-selected online sample who responded to advertisements published in pamphlets and posted on social media websites (e.g., Facebook) and websites and forums focused on pregnancy and childbirth (e.g., Rede Mãe, Forum PinkBlue). Advertisements about the study contained a web link to the online survey (hosted by http://www.limesurvey.com/). Participation was voluntary, and no remuneration was provided. Women were eligible to participate in the study if they were pregnant or had given birth during the last 12 months, and if they were 18 years or older. At the beginning of the survey, women were informed about the study's goals, assured of anonymity and confidentiality, and informed about the voluntary nature of their participation and the possibility of dropping out of the study at any time, without consequences. The participants provided their consent by answering a specific question regarding their willingness to participate in the study, and they were subsequently asked to complete the survey. The data were collected between April, 2014 and June, 2014.

\subsection{Measures}

An online self-report survey instrument was developed for this study, based on a literature review and similar surveys. The survey was pilot tested with a convenience sample ( $N=10$ women) and items were revised for clarity and comprehensibility.

The participants were asked to provide demographic (age, marital status, educational level, professional status, place of residence and family's household income) and clinical information: a) whether they were currently pregnant or had given birth during the last 12 months; b) parity (primiparity $v s$. multiparity); and c) medical history, including a history of psychiatric/psychological problems (yes vs. no) and a history of and current use of psychiatric/psychological treatment (yes vs.no). 
To assess the women's current use of online resources to address mental health issues, the participants were asked about the following: a) their knowledge ("Do you know of any websites related to illness/health topics?", yes vs. no) and frequency of use of websites concerning health/illness topics ("How frequently do you use the internet to obtain information about health/illness topcis?", from $1=$ Never to 6 = Daily); and b) their knowledge ("Do you know any website about mental health/emotional well-being?", yes vs. no) and frequency of use of websites concerning mental health/emotional well-being ("How frequently do you use the internet to obtain information about mental health/emotional well-being?", from $1=$ Never to $6=$ Daily). Moreover, to assess women's informational and participatory e-health behaviors pertaining to mental health issues, based in the study conducted by Chen and Lee [43], the participants were asked about their engagement ("Did you use the internet for...", answered on a dichotomous yes vs. no scale) in a variety of informational (4 items; e.g., "Search for emotional well-being, signs or symptoms of anxiety, depression or other psychological problems") and participatory e-health behaviors (5 items; e.g., "Share experiences online or find others who might share experiences concerning anxiety, depression, or other psychological problems in blogs, forums or Facebook)". Higher scores indicate the endorsement of a higher number of informational and participatory e-health behaviors.

To assess e-health literacy, the Portuguese version of the Ehealth Literacy Scale [44, 45] was used. The Ehealth Literacy Scale is a unidimensional scale comprising 8 items (e.g., "I know where to find helpful health resources on the internet"), answered on a Likert scale (from $1=$ Strongly Disagree to $5=$ Strongly Agree). Scale scores can range between 1 to 5, and higher scores indicate higher levels of ehealth literacy. This scale was developed to measure individuals' combined knowledge, comfort, and perceived skills at finding, evaluating, and applying electronic health information to health problems. The Portuguese version of the Ehealth Literacy Scale shows good psychometric properties [45]. In our sample, the Cronbach's alpha value for the Ehealth Literacy Scale items was .91.

The constructs of the C-TAM-TPB [31, 32] were used to assess women's acceptability-related perceptions of different types of e-mental health tools: 1) informative websites, 2) web-based psychological interventions, 3) online psychological appointments, and 4) online peer support groups. Specifically, based on the work of Ajzen [46] and on prior validated surveys assessing the same constructs of the C-TAM-TPB $[32,47,48]$, a set of items was developed to assess the acceptance and acceptability-related perceptions, concerning each of the e-mental health tool. Items were answered on a 5-point Likert scale (from $1=$ Strongly Disagree to $5=$ Strongly Agree). Higher scores indicate higher acceptance of e-mental health tools 
and more positive acceptability-related perceptions. Confirmatory Factor Analyses supported the construct validity of this measure to assess the constructs of the C-TAM-TPB for each of the e-mentalh health tools [Informative Websites: $\chi^{2}=95.60, \mathrm{p}<.001, \mathrm{CFI}=.96$, SRMR $=.038$; Web-based Psychological Interventions: $\chi^{2}=80.83, \mathrm{p}<.001, \mathrm{CFI}=.98, \mathrm{SRMR}=.024 ;$ Online Peer Support Groups: $\chi^{2}=77.75, \mathrm{p}<$ $.001, \mathrm{CFI}=.99, \mathrm{SRMR}=.021 ;$ Online Psychological Appointments: $\chi^{2}=118.10, \mathrm{p}<.001, \mathrm{CFI}=.98$, $\operatorname{SRMR}=.024]$.

Acceptance was inferred by the women's behavior intentions (the degree to which a person has formulated conscious plans to use the e-mental health tool), including 2 items: "I would resort to the $e$ mental health tool to help me deal with my psychological problems" and "I would recommend the e-mental health tool to a friend with psychological problems". Spearman-Brown correlations ranged from .60 [informative websites] to .82 [online psychologicaal appointments].

Acceptability-related perceptions, include the following [31]:

a)The individual's attitudes toward the behavior (positive or negative feelings about the e-mental health tool), including 2 items: "The e-mental health tool would be effective to address/alleviate my psychological problems" and "The e-mental health tool would be attractive". Spearman-Brown correlations ranged from .67 [informative websites] to .82 [online psychological appointments].

b)The individual's perceived behavioral control (perceptions of internal and external constraints on the use of the e-mental health tool), including 2 items: "The decision to use or not use the e-mental health tool would be mine" and "If I wanted, could to use the e-mental health tool". Spearman-Brown correlations ranged from .76 [web-based psychological interventions] to .80 [online peer support groups].

c)The individual's subjective norms (perceptions that most people who are important to the person think he should/should not use the e-mental health tool), with 1 item: "My family and/or friends would encourage me to use the e-mental health tool, to help me deal with my psychological problems".

d)The perceived usefulness (the degree to which an individual believes that using the e-mental health tool will help him), with 1 item: "The e-mental health tool would be useful for me".

e)The perceived ease of use (the degree of ease associated with the use of the e-mental health tool), with 1 item: "It would be difficult for me to use the e-mental health tool".

To assess the women's actual need for help (i.e., to screen for possible perinatal depressive symptoms), the Portuguese version of the EPDS [49-51] was used. The EPDS is a widely used 10-item scale that screens for antepartum and postpartum depressive symptoms. Although it has been originally 
developed to assess depressive symptoms in the postpartum period, it has also been proven to be a valid measure to assess depressive symptoms in pregnant women [52]. The women were asked to rate their emotions over the previous seven days concerning several symptoms (e.g., sadness, tearfulness, anxiety), using a 4-point Likert scale. In the Portuguese validation studies, a cutoff score higher than 9 is suggested to indicate possible clinically significant depressive symptoms [50-51]. In our sample, Cronbach's alpha value for the EPDS was .89. Based on the cutoff score, women were assigned to two groups: women with an actual need for help (i.e., EPDS positive group) and EPDS negative group.

\subsection{Data Analyses}

Statistical analyses were performed using the Statistical Package for the Social Sciences (IBM SPSS, v. 19). Descriptive statistics were used to characterize the sample's sociodemographic characteristics and to characterize the women's current patterns of use of online resources for mental health issues during the perinatal period. To compare the EPDS positive group and the EPDS negative group of women in their pattern of use of online resources for mental health issues during the perional period, we used chi-squared tests (to compare categorical variables: Knowledge of websites about health/illness and mental health topics, Frequency of use of websites about health/illness and mental health topics, Engagement in each type of informational and participatory e-health behavior) and t-tests (for continuous variables: Number of informational e-health behaviors, Number of participatory e-health behaviors, E-health literacy). Cohen's $d$ and Cramer's $V$ were used as effect-size measures. Moreover, repeated-measures ANOVAs were used to compare the women's acceptance (i.e., behavioral intention) and acceptability-related perceptions (attitudes toward the e-mental health tool, perceived behavioral control, ease of use, perceived usefulness and subjective norms), as a function of type of e-mental health tool (informative websites vs. web-based psychological interventions $v s$. online peer support groups $v s$. online psychological appointments; withinsubjects factor) and of group (EPDS positive group vs. EPDS negative group; between-subjects factor). Contrast analyses (Helmerth method; each condition is compared with the average effect of the subsequent conditions) were conducted.

Finally, four multiple linear regressions were performed, to identify the variables that most influence women's acceptance, i.e., women's intention to use each specific e-mental health tool. Effectsize measures were presented for the comparison analyses (small: $\eta^{2} \geq .01, d \geq .20, V=.01$; medium: $\eta^{2} \geq$ $.06, d \geq .50, V=.03 ;$ large: $\left.\eta^{2} \geq .14, d \geq .80, V=.05\right)$. The post-hoc power calculations conducted for the 
analyses performed with a significance level of .05 and a power $\geq .80$ indicated that small effects $(f=0.14$; $f^{2}=.02$ ) could be detected [53].

\section{Results}

\subsection{Participants}

A total of 546 women completed the survey. The sociodemographic and clinical characteristics of the sample are presented on Table 1 . The majority of women had given birth in the last 12 months $(M=$ 6.20 months, $S D=3.52$ months $)$, whereas $43.4 \%$ of women were currently pregnant $(M=24.22$ weeks pregnant, $S D=10.21$ weeks $)$. $24.0 \%$ of the women $(n=131)$ screened positive for depressive symptoms (EPDS positive group).

[Insert_Table_1_About_Here]

\subsection{Women's current pattern of use of online resources for mental health issues during the perinatal period}

Table 2 presents information concerning the women's knowledge and utilization of online resources regarding health/illness and mental health topics. The majority of women had prior knowledge of websites concerning health/illness topics $(n=443,81.1 \%)$, although their knowledge of websites focused on mental health topics was scarce $\left(n=171,31.3 \% ; \chi^{2}=35.49, p<.001\right.$, Cramer's $\left.V=.26\right)$. The EPDS positive group and the EPDS negative group of women reported a similar proportion of knowledge of websites pertaining to health/illness topics and mental health topics; however, EPDS positive women consulted those websites statistically significantly more frequently than EPDS negative women do (see Table 2).

\section{[Insert_Table_2_About_Here]}

Moreover, the women reported the use of informational and participatory e-health behaviors, as shown in Table 3. The women reported a statistically significantly higher number of informational e-health behaviors $(M=1.62, S D=1.45)$ than participatory e-health behaviors $\left(M=0.75, S D=1.02 ; t_{545}=15.12\right.$, $p<.001, d=.67)$. Compared with EPDS negative women, a significantly higher proportion of EPDS positive women have engaged in each informational and participatory e-health behavior, with the exception of sharing mental health-related content (e.g., news, videos) on social networks. EPDS positive women also 
reported a significantly higher number of informational and participatory e-health behaviors (see Tables 2 and 3).

[Insert_Table_3_about_here]

Finally, the women reported moderate levels of e-health literacy $(M=3.49, S D=0.75)$, and the levels were similar in both groups of women (see Table 2).

\subsection{Women's acceptance of e-mental health tools during the perinatal period}

\subsubsection{Comparison between different e-mental health tools and between EPDS positive women and EPDS negative women}

Table 4 presents women's acceptance and acceptability-related perceptions concerning the different e-mental health tools.

[Insert_Table_4_about_here]

There was a statistically significant effect of the type of tool in women's acceptance (intention to use), with women displaying more favorable intentions to use informative websites $\left(p<.001, \eta^{2}=.08\right)$ than the other tools. Additionally, a significant effect of group (EPDS positive $v s$. EPDS negative women) was found, with EPDS positive women reporting more favorable intentions to use the e-mental health tools. No interaction effect was found (see Table 4).

Moreover, statistically significant differences were found in all acceptability-related perceptions, with the exception of subjective norms (see Table 4). Specifically, the women were found to display more positive attitudes toward informative websites $\left(p<.001, \eta^{2}=.04\right)$ and a greater level of perceived behavioral control in using this e-mental health tool $\left(p<.001, \eta^{2}=.05\right)$ compared with the other tools. No significant differences were found between the remaining e-mental health tools concerning attitudes $\left(p=.377, \eta^{2}=\right.$ $.00)$ and behavioral control $\left(p=.271, \eta^{2}=.00\right)$. The women perceived informative websites as more useful than the remaining e-mental health tools $\left(p<.001, \eta^{2}=.19\right)$. Although no differences were found when comparing the perceived usefulness of web-based psychological interventions with the perceived usefulness of online peer support groups and online psychological appointments $\left(p=.608, \eta^{2}=.00\right)$, the women perceived the online peer support groups as more useful than online psychological appointments ( $p$ $\left.=.030, \eta^{2}=.01\right)$. Finally, the women perceived informative websites as easier to use than the other e-mental health tools $\left(p<.001, \eta^{2}=.11\right)$, followed by web-based psychological intervention programs $\left(p=.038, \eta^{2}\right.$ 
$=.01$ ), with no differences in the perceived easiness of use of online peer support groups and online psychological appointments $\left(p=.341, \eta^{2}=.00\right)$.

Additionally, EPDS positive women reported statistically significantly more positive attitudes toward the use of e-mental health tools and perceived them as more useful than EPDS negative women (see Table 4). Finally, no interaction effects were found in acceptability-related perceptions (see Table 4).

\subsubsection{Predictors of acceptance among EPDS positive women}

Table 5 presents the models predicting women's acceptance of (intentions to use) each e-mental health tool. The four models were statistically significant and explained between $30 \%$ and $89 \%$ of the variance in the predicted variables.

[Insert_Table_5_about_here]

As shown in Table 5, more favorable intentions to use informative websites, web-based psychological interventions and online psychological appointments were found in women who presented more positive attitudes toward the e-mental health tool, who perceived them as more useful and who had more positive perceptions of opinions from their social network (subjective norms). Perceived behavioral control over the use of the e-mental health tool and perceived ease of use significantly predicted the women's intention to use web-based psychological interventions and online psychological appointments, while marginally predicted the intention to use informative websites. By contrast, more positive attitudes toward the e-mental health tool were the only perception that significantly predicted the women's intention to use online peer support groups (see Table 5).

Moreover, the results showed that prior engagement in e-health behaviors significantly influenced the women's intention to use web-based psychological interventions and online peer support groups: specifically, greater engagement in participatory e-health behaviors and higher levels of e-health literacy were significant predictors of the women's intention to use web-based psychological interventions, while greater engagement in informational e-health behaviors significantly predicted the women's intention to use online peer support groups. 


\section{Discussion}

The prevalent and disabling nature of mental health problems has led to the increasing global trend to utilize electronic media to enhance public access to mental health information, self-help strategies, and prevention or treatment services [54]. Given women's low help-seeking rates for mental health problems during the perinatal period $[11,55]$, the use of digital technologies to provide mental health information and treatment may be a valuable option. However, it still remains important to establish the acceptability of such tools in the perinatal population, particularly in countries that have not yet developed these e-mental health tools, such as Portugal.

Although exploratory, the results of the present study provide some evidence supporting women's current utilization of online resources to address health and mental health concerns. First, our results showed that the majority of the women in our sample consult websites focused on health/illness topics at least several times per year, which is congruent with prior studies [e.g., 16]. Second, there is also some evidence of women's utilization of online resources addressing mental health topics during the perinatal period. Specifically, the majority of women have already engaged in at least one type of informational or participatory e-health behavior related to mental health, particularly in searching for signs of psychological problems and for self-help strategies. These results are consistent with the findings of Maloni et al. [17], who found that a significant proportion of women searched the internet for information about depressive symptoms during the perinatal period and found the information helpful.

Third, the results of this study are particularly innovative because they identify differences in the use of online resources among women with a positive screen for depressive symptoms and women with a negative screen. Women presenting an actual need for help seem to be more likely to use online resources to deal with their mental health problems. In a recent study, Chen and Lee [43] found an inverse association between the levels of mental health problems and the number of informational e-health behaviors, with no relationship between physical health and e-health behaviors. These results seem to support the potential utility of the internet for the delivery of accurate mental healthcare during the perinatal period. The use of online information may assist women presenting an actual need for help to better understand their mental health condition, to explore treatment options or to complement the information given by health professionals (informational behaviors), in addition to feeling less isolated and less stigmatized by sharing their experiences with others (participatory behaviors; [17, 18]). 
Moreover, the results provide some interesting insights on Portuguese women's acceptance of emental health tools. The average scores on the acceptance and acceptability-related perceptions suggest good acceptability of e-mental health tools among Portuguese women. However, and although these results should be cautiously interpreted due to the magnitude of size effects, women who present an actual need for help tend to display more favorable intentions to use e-mental health tools, as well as more positive perceptions of such tools (positive attitudes and greater perceived usefulness). Those women constitute the target population for potential prevention and/or treatment services aiming to alleviate perinatal distress symptoms. Therefore, these results support the suitability of e-mental health tools and the relevance of developing empirically driven and culturally adapted tools for addressing perinatal distress in Portugal.

Additionally, the results showed that informative websites are more acceptable to women than the other e-mental health tools are, as they show more positive attitudes toward these websites and perceive them as more useful, easy to use, and having fewer constraints on their use. One possible explanation for these results may be the greater contact of this population with informative websites than with other tools. In fact, our results showed that while approximately $60 \%$ of women have searched for mental health information online, only approximately $19 \%$ have shared their experience online (e.g., through online peer support groups) and a minority have had online psychological appointments. Moreover, there have been no Portuguese web-based psychological interventions targeting perinatal distress. In fact, consistent with this hypothesis, prior studies have shown that greater acceptance of e-mental health interventions is associated with the prior use of such interventions [38, 39].

Finally, our results globally contribute to the empirical validation of C-TAM-TPB theory [31,32], by showing that women's intentions to use most e-mental health tools can be predicted by the different acceptability-related perceptions (attitudes toward the e-mental health tool, perceived behavioral control, perceived usefulness and ease of use, and subjective norms). Therefore, to improve the acceptance of emental health tools by women who present an actual need for help, efforts should be directed toward changing women's acceptability-related perceptions, as discussed below. However, it is important to note that apart from attitudes toward the e-mental health tool, no other construct of the C-TAM-TPB significantly contributed to explain women's intention to use online peer support groups to help them with mental health difficulties during the perinatal period. One possible explanation may be related to the fact that online peer support groups are characterized by interactivity among members (sharing experiences with others undergoing similar psychological experiences), which is a distinctive feature of this e-mental 
health tool. Therefore, it is possible that the women's availability for engaging in this type of behavior may be dependent on other variables that influence help-seeking in general, such as psychological openness to experience [56]. This hypothesis should be further explored.

Taken together, the results of this study seem to globally support Portuguese women's acceptance of e-mental health tools during the perinatal period. Although these findings can be generalized only to the Portuguese population, they may provide some indications of the acceptance of e-mental health tools in countries presenting similar levels of new technology penetration and similar levels of development of emental health tools. However, the limitations of this study must also be acknowledged. First, this study was subject to selection bias, because internet survey participants are limited to those who use the internet (that is, the perspective of those women who do not use the internet, and may be less prone to e-mental health tools, is absent). Future studies on this topic should also consider other forms of recruitment, namely faceto-face recruitment at perinatal health services, in order to overcome this limitation. Second, the internet survey was based on voluntary responses; people who were willing to participate in this study may be more accepting of the use of digital technologies as a channel for mental health care delivery. Despite this possible bias, the response to our study recruitment was very large, suggesting that a significant population of women in the perinatal period may have internet access and may be willing to engage in e-mental health tools, when needed. Third, the constructs of the C-TAM-TPB theory were assessed through a measure spefically developes by the authors, based on prior surveys with similar goals [32, 47-48], which may compromise the interpretation of the results. Fourth, in the present study we did not compare the acceptance of e-mental health tools with the acceptance of face-to-face treatments.

In conclusion, when considering the development of new e-mental health tools targeting perinatal distress for the Portuguese population, the results of this study should be taken into account. The development of informative websites seems to have ensured greater acceptance by the target population. Thus, the focus on developing empirically based informative websites in the initial stage of the development of new e-mental health tools targeting perinatal distress may increase rates of successful implementation and outcomes. Moreover, when considering the development of other e-mental health tools (e.g., web-based psychological interventions, online psychological appointments), two different types of strategies can be implemented. First, women should be involved as stakeholders in the process of developing such e-mental health tools, so that the characteristics of these tools improve women's level of acceptance. Second, when disseminating the e-mental health tools, women should be provided with information that may help them 
to change their existing perceptions, thus improving the acceptance of such tools. Specifically, dissemination actions directed toward potential users should include not only information about the benefits, costs and efficacy results of the e-mental health tools but also the opportunity to experiment with the tools and to clarify doubts about their use.

Finally, the results of this study seem to be encouraging of the integration of e-mental health tools in a stepped-care treatment model for perinatal depression [57]. As mentioned by prior studies [58], the advent of technology makes the use of stepped-care much more viable in clinical practice. In Portugal, no screening procedures are implemented to improve case identification and early interventions, and mental healthcare is provided only when women seek it, or when health professionals identify symptoms worthy of clinical attention. Therefore, future studies should seek to understand how e-mental health tools can complement existing screening, diagnosis, and treatment resources for perinatal depression.

\section{References}

[1] European Comission (2014). eHealth Action Plan 2012-2020: Innovative healthcare for the 21st century. European Comission: Brussels.

[2] Hogan, T., Wakefield, B., Nazi, K., Houston, T., \& Weaver, F. (2011). Promoting access through complementary ehealth technologies: Recommendations for VA's home telehealth and personal health record programs. Journal of General Internal Medicine, 26, 628-635. doi: 10.1007/s11606$011-1765-y$

[3] Lal, S., \& Adair, C. (2014). E-mental health: A rapid review of the literature. Psychiatric Services, 65, 24-32. doi:10.1176/appi.ps.201300009

[4] Christensen, H., Griffiths, K., \& Evans, K. (2002). E-mental health in Australia: Implications of the Internet and related technologies for policy. Canberra: Commonwealth Department of Health and Ageing.

[5] Riper, H., Andersson, G., Christensen, H., Cuijpers, P., \& Lange, A. (2010). Theme issue on e-mental health: A growing field in internet research. Journal of Medical Internet Research, 12(5), e74. doi: 10.2196/jmir.1713

[6] Casey, L., Joy, A., \& Clough, B. (2013). The impact of information on attitudes toward e-mental health services. CyberPsychology, Behavior and Social Networking, 16(8), 593-598. doi:10.1089/cyber.2012.0515 
[7] Gaynes, B., Gavin, N., Meltzer-Brody, S., Lohr, K., Swinson, T., Gartlehner, G., . . Miller, W. (2005). Perinatal depression: Prevalence, screening accuracy and screening outcomes Evidence Report/Technology Assessment (Vol. 119). RockVille, MD: Agency for Healthcare Research and Quality.

[8] Muzik, M., \& Borovska, S. (2010). Perinatal depression: Implications for child mental health. Mental Health in Family Medicine, 7, 239-247.

[9] Kingston, D., Tough, S., \& Whitfield, H. (2012). Prenatal and postpartum maternal psychological distress and infant development: a systematic review. Child Psychiatry \& Human Development, 43(5), 683-714. doi: 10.1007/s10578-012-0291-4

[10] Tronick, E., \& Reck, C. (2009). Infants of depressed mothers. Harvard Review of Psychiatry, 17(2), 147-156. doi: 10.1080/10673220902899714

[11] Dennis, C. L., \& Chung-Lee, L. (2006). Postpartum Depression help-seeking barriers and maternal treatment preferences: A qualitative systematic review. Birth, 33, 323-331. doi: 10.1111/j.1523536X.2006.00130.x

[12] O'Mahen, H. A., \& Flynn, H. A. (2008). Preferences and perceived barriers to treatment for depression during the perinatal period. Journal of Women's Health, 17(8), 1301-1309. doi: 10.1089/jwh.2007.0631

[13] Danaher, B. G., Milgrom, J., Seeley, J. R., Stuart, S., Schembri, C., Tyler, M. S., . . Lewinsohn, P. (2012). Web-Based Intervention for Postpartum Depression: Formative Research and Design of the MomMoodBooster Program. Journal of Medical Internet Research, 1(2), e18. doi: 10.2196/resprot.2329

[14] Haga, S., Drozd, F., Brendryen, H., \& Slinning, K. (2013). Mamma mia: A feasibility study of a webbased intervention to reduce the risk of postpartum depression and enhance subjective well-being. Journal of Medical Internet Research, 2, e29. doi: 10.2196/resprot.2659

[15] Moore, D., \& Ayers, S. (2011). A review of postnatal mental health websites: Help for healthcare professionals and patients. Archives of Women's Mental Health, 14, 443-452. doi:10.1007/s00737011-0245-Z

[16] Fleming, S. E., Vandermause, R., \& Shaw, M. (2014). First-time mothers preparing for birthing in an electronic world: internet and mobile phone technology. Journal of Reproductive and Infant Psychology, 32(3), 240-253. doi: 10.1080/02646838.2014.886104 
[17] Maloni, J. A., Przeworski, A., \& Damato, E. G. (2013). Web recruitment and internet use and preferences reported by women with postpartum depression after pregnancy complications. Archives of Psychiatric Nursing, 27(2), 90-95. doi: 10.1016/j.apnu.2012.12.001

[18] Evans, M., Donelle, L., \& Hume-Loveland, L. (2012). Social support and online postpartum depression discussion groups: A content analysis. Patient Education and Counseling, 87(3), 405-410. doi: 10.1016/j.pec.2011.09.011

[19] Barrera, A., Wickham, R., \& Muñoz, R. (2015). Online prevention of postpartum depression for Spanish- and English-speaking pregnant women: A pilot randomized controlled trial. Internet interventions, 2, 257-265. doi:10.1016/j.invent.2015.06.002

[20] Danaher, B. G., Milgrom, J., Seeley, J. R., Stuart, S., Schembri, C., Tyler, M. S., . . Lewinsohn, P. (2013). MomMoodBooster web-based intervention for postpartum depression: Feasibility Trial Results. Journal of Medical Internet Research, 15(11), e242. doi:10.2196/jmir.2876

[21] O’Mahen, H. A., Woodford, J., McGinley, J., Warren, F. C., Richards, D., Lynch, T., \& Taylor, R. S. (2013). Internet-based behavioral activation treatment for postnatal depression (Netumums): A randomized controlled trial. Journal of Affectice Disorders, 150(3), 814-822. doi:10.1016/j.jad.2013.03.005.

[22] Pugh. N., Hadjistavropoulos, H., Hamptom, A., Bowen, A., \& Williams, J. (2015). Client experiences of guided internet cognitive behavior therapy for postpartum depression: A qualitative study. Archives of Women's Mental Health, 18, 209-219. doi:10.1007/s00737-014-0449-0

[23] Richards, D., \& Richardson, T. (2012). Computer-based psychological treatments for depression: a systematic review and meta-analysis. Clinical Psychology Review, 32(4), 329-342. doi: 10.1016/j.cpr.2012.02.004

[24] Musiat, P., Goldstone, P., \& Tarrier, N. (2014). Understanding the acceptability of e-mental health: Attitudes and expectations towards computerised self-help treatments for mental health problems. Psychiatry, 14, 109. doi: 10.1186/1471-244x-14-109

[25] Waller, R., \& Gilbody, S. (2009). Barriers to the uptake of computerized cognitive behavioural therapy: A systematic review of the quantitative and qualitative evidence. Psychological Medicine, 39, 705-712. doi: 10.1017/S0033291708004224 
[26] Farrer, L., Gulliver, A., Bennett, K., \& Griffiths, K. (2015). Exploring the acceptability of online mental health interventions among university teaching staff: Implications for intervention dissemination and uptake. Internet Interventions, 2, 359-365. doi:10.1016/j.invent.2015.07.006

[27] Hardiker, N., \& Grant, M. (2011). Factors that influence public engagement with ehealth: A literature review. International Journal of Medical Informatics, 80, 1-12. doi: 10.1016/j.ijmedinf.2010.10.017

[28] Hofstede, J., de Bie, J., Wijngaarden, v., \& Heijmans. (2014). Knowledge, use and attitudes toward eHealth among patients with chronic lung diseases. International Journal of Medical Informatics, 83(12), 967-974. doi: 10.1016/j.ijmedinf.2014.08.011

[29] Hardiker, N., \& Grant, M. (2011). Factors that influence public engagement with eHealth: A literature review. International Journal of Medical Informatics, 80(1), 1-12. doi: 10.1016/j.ijmedinf.2010.10.017

[30] Gattiker, U. E. (1984). Managing computer-based office information technology: A process model for management. In H. Hendrick \& O. G. Brown (Eds.), Human factors in organizational design (pp. 395-403). The Netherlands: Elsevier Science.

[31] Taylor, S., \& Todd, P. (1995). Assessing IT Usage: The role of prior experience. Management Information Systems Quarterly, 19(4), 561-570.

[32] Venkatesh, V., Morris, M., Davis, G., \& Davis, F. (2003). User acceptance of information technology: Toward a unified view. Management Information Systems Quarterly, 27(3), 425-478.

[33] Wootton, B., Titov, N., Dear, B. F., Spence, J., \& Kemp, K. (2011). The acceptability of internet-based treatment and characteristics of an adult sample with obsessive compulsive disorder: An internet survey. PLoS One, 6, e20548. doi: 10.1371/journal.pone.0020548

[34] Carper, M., McHugh, R., \& Barlow, D. (2013). The dissemination of computer-based psychological treatment: A preliminary analysis of patient and clinician perceptions. Administration and Policy in Mental Health and Mental Health Services Research, 40, 87-95. doi: 10.1007/s10488-0110377-5

[35] Young, K. (2005). An empirical examination of client attitudes towards online counseling. Cyberpsychology \& Behavior, 8(2), 172-177. 
[36] Chang, T., \& Chang, R. (2004). Counseling and the internet: Asian-American and Asian-International college students' attitudes toward seeking online professional psychological help. Journal of College Counseling, 7, 140-149. doi:10.1002/j.2161-1882.2004.tb00245.x

[37] Rochlen, A., Beretvas, N., \& Zack, J. (2004). The online and face-to-face counseling attitudes scales: A validation study. Measurement and Evaluation in Counseling and Development, 37, 95-111.

[38] Klein, B., \& Cook, S. (2010). Preferences for e-mental health services amongst an online Australian sample. Electronic Journal of Applied Psychology, 6(1), 27-38.

[39] Gun, S., Titov, N., \& Andrews, G. (2011). Acceptability of internet treatment of anxiety and depression. Australian Psychiatry, 19(3), 259-264. doi: 10.3109/10398562.2011.562295

[40] Khazaal, Y., Chatton, A., Cochand, S., Hoch, A., Khankarli, M. B., Khan, R., \& Zullino, D. F. (2008). Internet use by patients with psychiatric disorders in search for general and medical informations. Psychiatry Quarterly, 79(4), 301-309. doi: 10.1007/s11126-008-9083-1

[41] World Medical Association (2000). Declaration of Helsinki: Ethical principles for medical research involving human subjects. Journal of the American Medical Association, 284, 3043-3045. doi:10.1001/jama.284.23.3043

[42] American Psychological Association (2010). Publication manual of the American Psychological Association (6 $6^{\text {th }}$ ed.). London: American Psychological Association.

[43] Chen, W., \& Lee, K.-H. (2014). More than search? Informational and participatory eHealth behaviors. Computers in Human Behavior, 30, 103-109. doi: 10.1016/j.chb.2013.07.028

[44] Norman, C., \& Skinner, H. (2006). eHEALS: The eHealth Literacy Scale. Journal of Medical Internet Research, 8(4), e27. doi: doi:10.2196/jmir.8.4.e27

[45] Tomás, C., Queirós, P., \& Ferreira, T. (2014). Análise das propriedades psicométricas da versão portuguesa de um instrumento de avaliação de e-Literacia em Saúde [Analysis of psychometric properties of the portuguese version of an eHealth literacy assessment tool]. Revista de Enfermagem Referência, 2, 19-28. doi:10.12707/RIV14004

[46] Ajzen, I. (2002). Construction of a standard questionnaire for the theory of planned behavior. Retrieved April 12, 2014, from http://www-unix.oit.umass.edu/ aizen.

[47] Safeena, R., Date, H., Hundewale, N., \& Kammani, A. (2013). Combination of TAM and TPB in Internet banking adoption. International Journal of Computer Theory and Engineering, 5(1), 146150. doi:10.7763/IJCTE.2013.V5.665 
[48] Tan, P. (2013). Applying the UTAUT to understand factors affecting the use of English e-learning websites in Taiwan. SAGE open, October-December, 1-12. doi:10.1177/2158244013503837

[49] Cox, J., Holden, J. M., \& Sagovsky, R. (1987). Detection of postnatal depression: Development of the 10-item Edinburgh Postnatal Depression Scale. British Journal of Psychiatry, 150, 782-786. doi: 10.1192/bjp.150.6.782

[50] Areias, M., Kumar, R., Barros, H., \& Figueiredo, E. (1996). Comparative incidence of depression in women and men, during pregnancy and after childbirth: Validation of the Edinburgh Postnatal Depression Scale in Portuguese Mothers. British Journal of Psychiatry, 169(1), 30-35. doi: 10.1192/bjp.169.1.30

[51] Augusto, A., Kumar, R., Calheiros, J., Matos, E., \& Figueiredo, E. (1996). Post-natal depression in an urban area of Portugal: Comparison of childbearing women and matched controls. Psychological Medicine, 26, 135-141. doi: 10.1017/S0033291700033778

[52] McBride, H., Wiens, R., McDonald, M., Cox, D., \& Chan, E. (2014). The Edinburgh Postnatal Depression Scale (EPDS): A review of the reported validity evidence. In B. D. Zumbo \& E. K. Chan (eds.), Validity and validation in Social, Behavioral, and Health Sciences. Switzerland: Springer International. doi:10.1007/978-3-319-07794-9_9

[53] Faul, F., Erdfelder, E., Lang, A.-G., \& Buchner, A. (2007). G*Power 3: A flexible statistical power analysis program for the social, behavioral, and biomedical sciences. Behavior Research Methods, $39,175-191$.

[54] Harrison, V., Proudfoot, J., Wee, P., Parker, G., Pavlovic, D., \& Manicavasagar, V. (2011). Mobile mental health: Review of the emerging field and proof of concept study. Journal of Mental Health, 20(6), 509-524. doi: 10.3109/09638237.2011.608746

[55] Fonseca, A., Gorayeb, R., \& Canavarro, M. C. (2015). Women's help-seeking behaviours for depressive symptoms during the perinatal period: Socio-demographic and clinical correlates and perceived barriers to seeking professional help. Midwifery, 12, 1177-1185. doi:10.1016/j.midw.2015.09.002

[56] Mackenzie, C., Knox, V., Gekoski, W., \& Macaulay, H. (2004). An adaptation and extension of the Attitudes Toward Seeking Professional Psychological Help Scale. Journal of Applied Social Psychology, 34(11), 2410-2433. doi: 10.1111/j.1559-1816.2004.tb01984.x 
[57] Gjerdingen, D., Crow, S., McGovern, P., Miner, M., \& Center, B. (2009). Stepped care treatment of postpartum depression: Impact on treatment, health and work outcomes. The Journal of the American Board of Family Medicine, 22(5), 473-482. doi:10.3122/jabfm.2009.05.080192

[58] Mental Health Comission of Canada (2014). E-mental health in Canada: Transforming the mental health system using technology. Ottawa, ON: Mental Health Comission of Canada. Retrieved from: http://www.mentalhealthcomission.ca 
Table 1. Sociodemographic and clinical characteristics of the sample

Women $(N=546)$

\section{Sociodemographic characteristics}

Age (in years), $M(S D)$

$31.55(4.09)$

Educational level, $n(\%)$

Middle school

High school

Higher education
$13(2.4)$

$110(22.5)$

$423(77.5)$

Marital status, $n(\%)$

Single $50(9.2)$

Married/living together

$492(90.1)$

Separated/divorced

$4(0.7)$

Professional status, $n(\%)$

$$
\text { Employed }
$$

Unemployed

Other

$17(3.3)$

Monthly Household income, $n(\%)$

$$
\begin{array}{r}
<500 € \\
500-1,000 € \\
1,000-2,000 € \\
2,000-3,500 € \\
>3,500 €
\end{array}
$$

$131(24.0)$

$30(5.5)$

Residence, $n(\%)$
$458(83.9)$

$88(16.1)$

\section{Clinical characteristics}


Pregnancy

Postpartum period

Parity, $n(\%)$
$237(43.4)$

$309(56.6)$

$382(70.0)$

Multiparity

$164(30.0)$

Psychiatric history, $n(\%)$

History of psychiatric/psychological $141(25.8)$

problems (Yes)

History of psychiatric/psychological

$131(25.6)$

treatment (Yes) 
Table 2. Current pattern of use of online resources for health and mental health issues among EPDS positive women and EPDS negative women during the perinatal period.

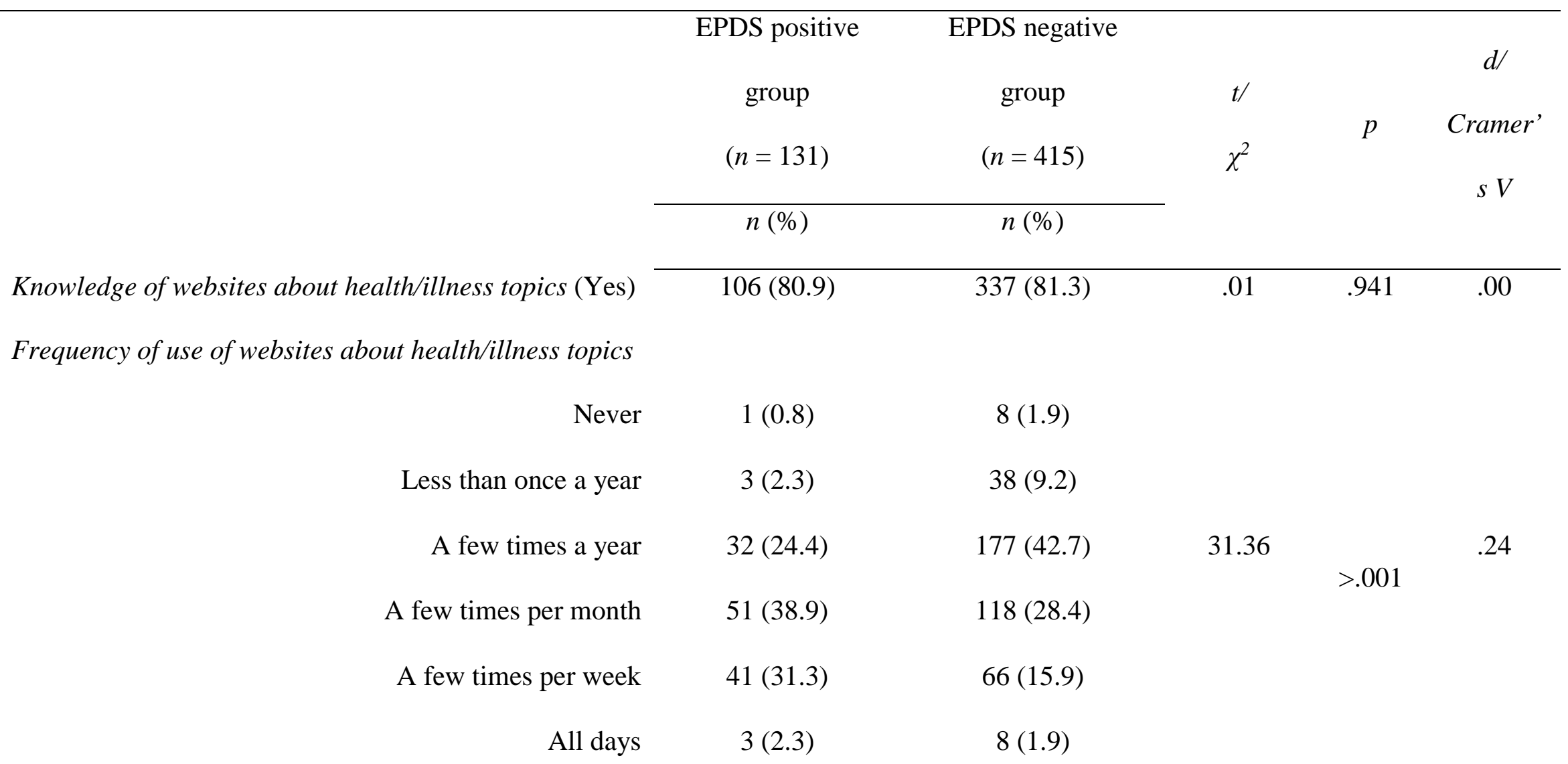


Frequency of use of websites about mental health topics

\begin{tabular}{|c|c|c|c|c|c|}
\hline Never & $24(18.3)$ & $139(33.5)$ & & & \\
\hline Less than once a year & $18(13.7)$ & $126(30.4)$ & & $>.001$ & .30 \\
\hline A few times a year & $49(37.4)$ & $102(24.6)$ & 50.56 & & \\
\hline A few times per month & $22(16.8)$ & $31(7.5)$ & & & \\
\hline A few times per week & $17(13.0)$ & $13(3.1)$ & & & \\
\hline \multirow[t]{3}{*}{ All days } & $1(0.8)$ & $4(1.0)$ & & & \\
\hline & $M(S D)$ & $M(S D)$ & & & \\
\hline & $2.27(1.43)$ & $1.41(1.39)$ & 6.13 & $>.001$ & .61 \\
\hline$h$ behaviors & $1.13(1.21)$ & $0.63(0.93)$ & 4.39 & $>.001$ & .46 \\
\hline & $3.39(0.80)$ & $3.52(0.73)$ & -1.67 & .096 & .17 \\
\hline
\end{tabular}

(Range: 1-5) 
Table 3. Engagement in informational and participatory e-health behaviors among depressed and non-depressed women during the perinatal period

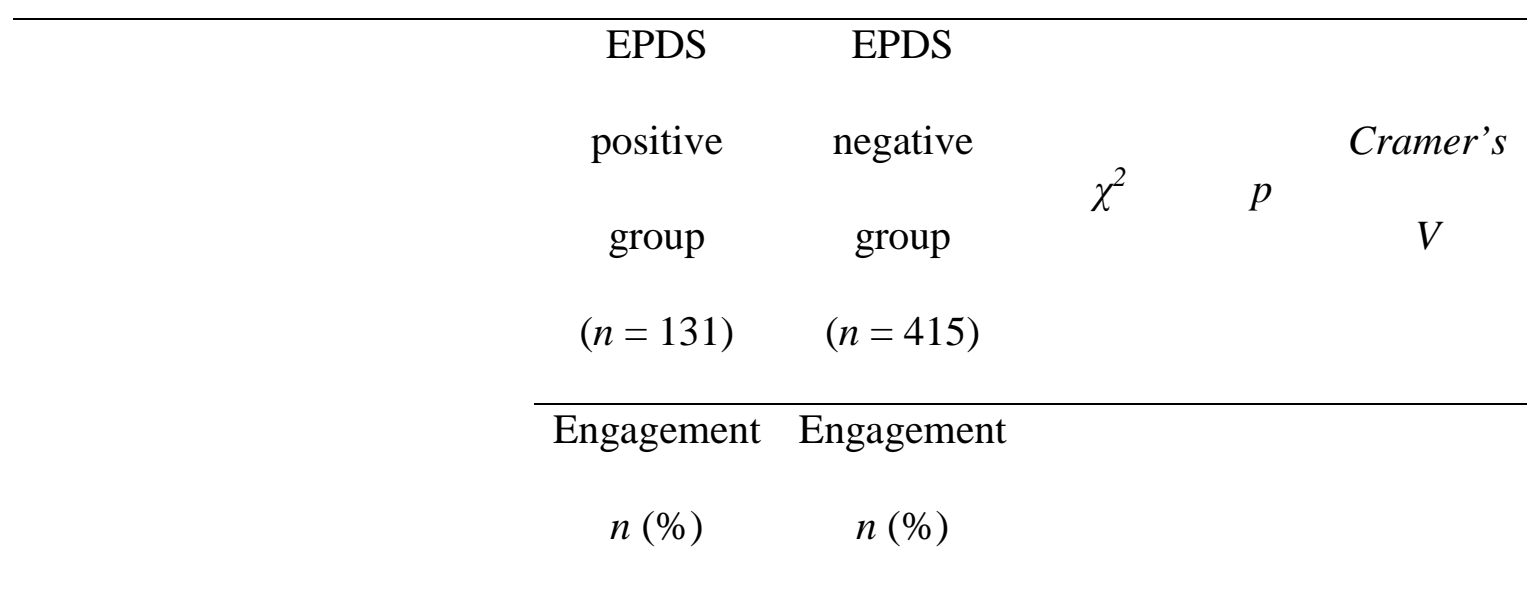

Informational e-health behaviors (Range: 0-4)

Search for emotional well-

being, signs or symptoms of

$102(77.9) \quad 225(54.2) \quad 23.18 \quad<.001 \quad .21$

anxiety, depression or other

psychological problems

Search for treatment options

for anxiety, depression or

other psychological

problems (including

$68(51.9) \quad 115(27.7) \quad 26.16 \quad<.001$

medical, psychological or

alternative medicine

treatments)

Search for health

professionals (e.g.,

psychologists, psychiatrists)

$\begin{array}{lllll}51(38.9) & 99(23.9) & 11.36 & .001 & .14\end{array}$

or places (e.g., hospitals, 
clinical practices) where

treatment for psychological

problems can be found

Search for strategies to

promote emotional well-

being or self-help strategies

$\begin{array}{lllll}77(58.8) & 148(35.7) & 21.96 & <.001 & .20\end{array}$

to deal with anxiety,

depression or other

psychological problems

Participatory e-health behaviors (Range: 0-5)

Share news, photos, videos

or audio files about

emotional well-being or

$22(16.8) \quad 70(16.9)$

$.00 \quad .984$

.00

psychological problems in

social networks

Share experiences online or

find others that might share

experiences concerning

anxiety, depression, or other

$\begin{array}{lllll}43(32.8) & 59(57.8) & 22.69 & <.001 & .20\end{array}$

psychological problems in

blogs, forums, facebook

Interact with mental health

professionals (e.g.,

psychologists or

$\begin{array}{lllll}15(11.5) & 18(4.3) & 8.87 & .003 & .13\end{array}$

psychiatrists) to clarify 
doubts through email, chat,

forums or other platforms

Assess emotional well-being

(e.g., filling out

questionnaires on

$61(46.6) \quad 108(26.0) \quad 19.66 \quad<.001$

.19

depression, anxiety, stress,

personality traits)

Have online appointments

$.005 \quad .12$

(e.g., via Skype, chat) with

$7(5.3)$

$5(1.2)$

7.93

mental health professionals 
Table 4. Women's acceptance of different e-mental health tools: Acceptance and acceptability-related perceptions among EPDS positive women and EPDS negative women.

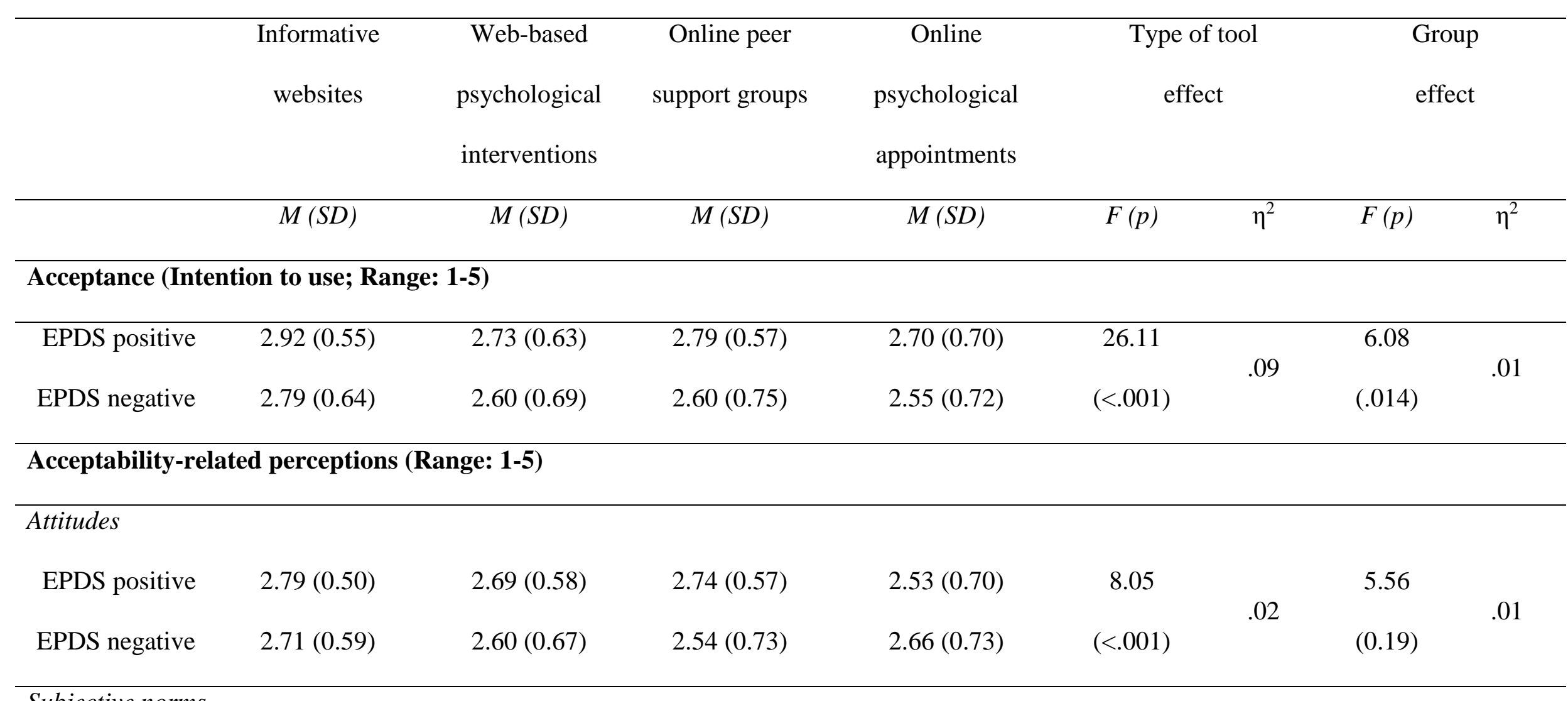

Subjective norms 


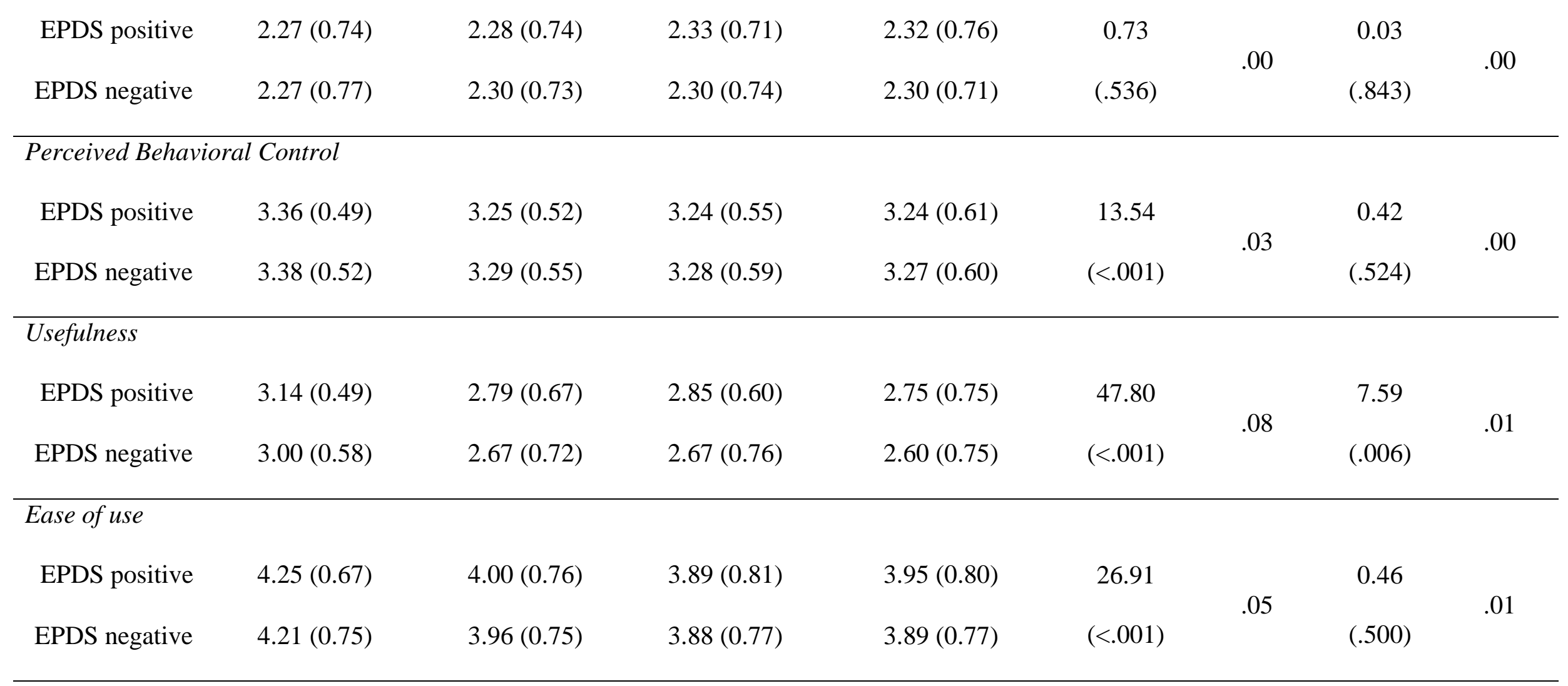


Table 5. Acceptance predictors of e-mental health tools.

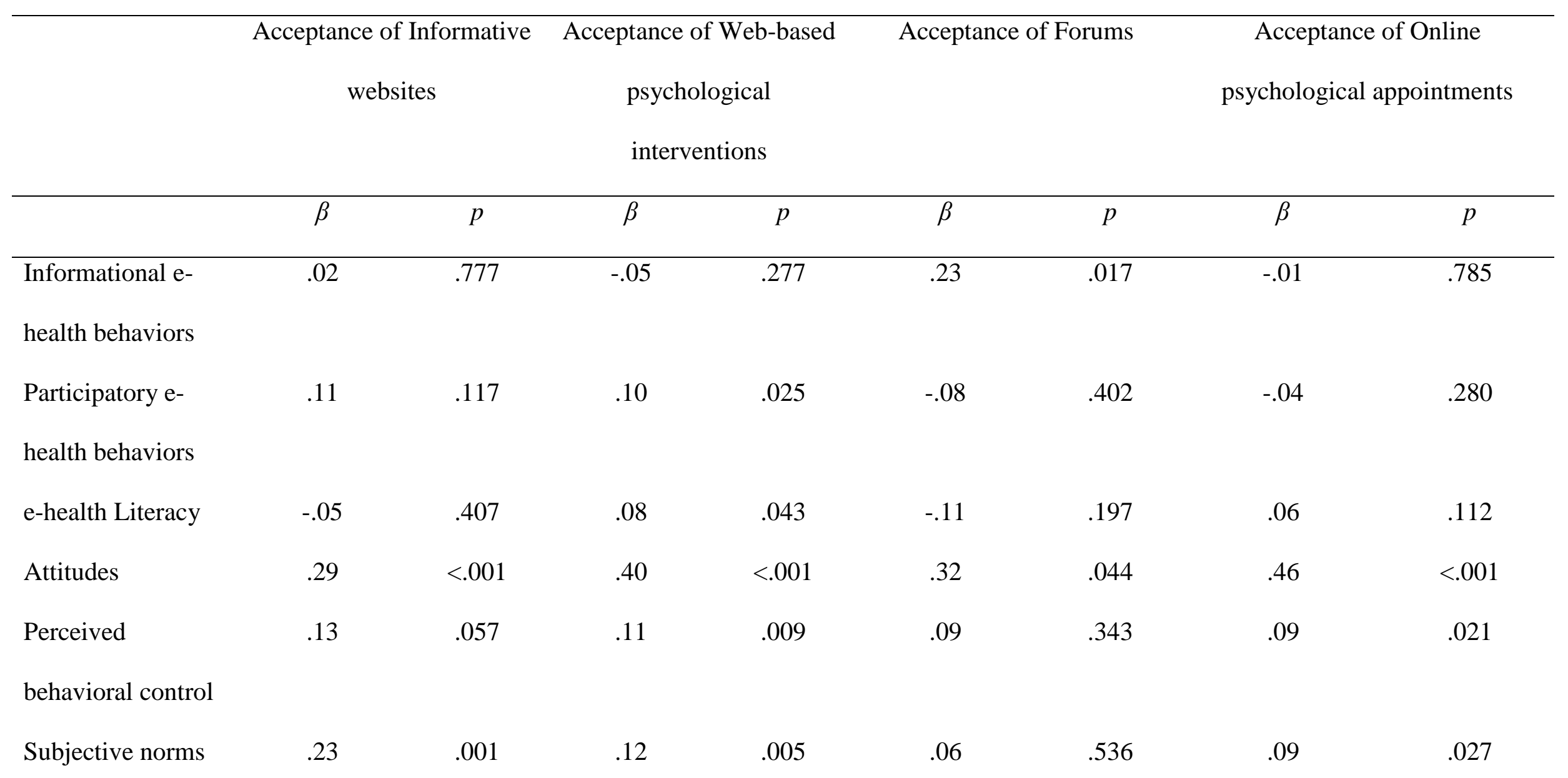




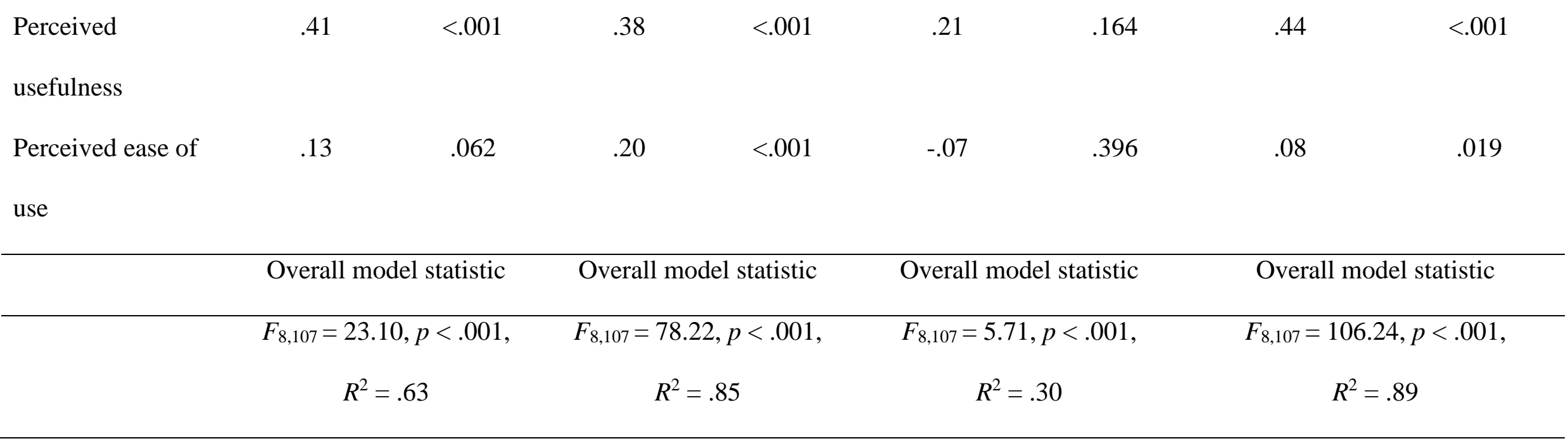

